\title{
A REMARK ON SET THEORY
}

\section{ARLEN BROWN}

In verifying the equivalence of the familiar triad: (1) the Axiom of Choice, (2) Zermelo's well-ordering theorem, and (3) the Maximum Principle, the only difficulties that arise lie in deriving (2) and (3) from (1). The two constructions of Zermelo [3], [4] provide derivations of (2) from (1), and it is not hard to see that the second of Zermelo's proofs can be modified so as to show that (1) also implies (3) (see, for instance $[2, \S 16]$ ). For reasons of economy and symmetry it is desirable to isolate the common features of these two derivations. The purpose of this note is to indicate how a single elementary construction can be used to prepare the way for both at once. Let $X$ be a nonempty, partially ordered set with the property that every nonempty, simply ordered subset $J$ of $X$ has a supremum $\sup J$ in $X$, and suppose given a mapping $f: X \rightarrow X$ with the property that $f(x) \geqq x$ for every $x$ in $X$. We shall say that a subset $K$ of $X$ is an $f$-tower if (i) $f(K) \subset K$, and if (ii) whenever $J$ is a nonempty, simply ordered subset of $K$ we have sup $J \in K$. Our main result is then as follows.

Lemma. If $X$ and $f$ are as stated, then there exist well-ordered $f$-towers. Indeed, for each element $x$ of $X$ there exists a well-ordered $f$-tower containing $x$.

This result, which does not itself require the Axiom of Choice, and for which a proof can readily be modeled after $[2$, loc. cit], is obtained by crossing Zermelo's construction [4] with a lemma due to Bourbaki (see below), and contains essentially nothing new. Its advantage lies in the fact that it leads to the desired results with optimum efficiency. In the first place, we have

Theorem 1 (Bourbaki [1, p. 37]). Under the same hypotheses as those of the lemma, $f$ possesses a fixed point.

Proof. Let $K$ be a nonempty, simply ordered $f$-tower. Then $s=\sup K \in K$, and therefore $f(s) \leqq s$.

Next, getting down to business, we have

Theorem 2 (Zermelo [3], [4]). The Axiom of Choice implies the well-ordering theorem.

Proof. (This is precisely Zermelo's second argument [4].) Let $A$

Received by the editors October 14, 1969. 
be a set. Using a choice function, it is easy to assign to each subset $E$ of $A(E \neq A)$ a distinguished element $c(E)$ of the complement $A \backslash E$. Then

$$
\begin{aligned}
f(E) & =E \cup\{c(E)\}, & & E \neq A, \\
& =A, & & E=A
\end{aligned}
$$

defines a map with the property $F(E) \supset E$ on the system $x$ of all subsets $E$ of $A$, and by the lemma there exists a well-ordered nest $\Re$ of subsets that begins with $\varnothing$ and that is an $f$-tower (with respect to $C$ ). Since $\mathscr{K}$ must end with a fixed point of $f$, we have $A \in \mathscr{K}$ and therefore, for each $a \in A$, there exists a smallest set $E \in \mathcal{K}$ such that $a \in E$. Denote this set by $E(a)$, and define $a$ to be $\leqq b$ when and only when $E(a) \subset E(b)$. Using the fact that $\mathcal{K}$ is well ordered, it is easy to see that the mapping $a \rightarrow E(a)$ is one-one, and hence that $\leqq$ well orders $A$.

Theorem 3. The Axiom of Choice implies the Maximum Principle.

Proof. It suffices to show that any partially ordered set $X$ contains a maximal simply ordered subset. Suppose the contrary. Then, using a choice function, we may assign to each simply ordered subset $J$ of $X$ a simply ordered subset $c(J)$ such that $c(J) \supset J$ and $c(J) \neq J$. Since the system $\delta$ of all simply ordered subsets of $X$ satisfies the hypotheses of the lemma, this contradicts Theorem 1.

\section{REFERENCES}

1. N. Bourbaki, Thêorie des ensembles (Fascicule de résultats), Actualités Sci. Indust., no. 846, Hermann, Paris, 1939. MR 3, 55.

2. P. R. Halmos, Naive set theory, The University Series in Undergraduate Math., Van Nostrand, Princeton, N.J., 1960. MR 22 \#5575.

3. E. Zermelo, Beweis, dass jede Menge wohlgeordnet werden kann, Math. Ann. 59 (1904), 514-516.

4. - Neuer Beweis für die Möglichkeit einer Wohlordnung, Math. Ann. 65 (1908), 106-128.

INDIANA UNIVERSITY, BLOOMINGToN, INDIANA 47401 